UDC 539

\title{
Few-body neutron and kaonic clusters
}

\author{
Kezerashvili R.Ya., ${ }^{1,2}$ \\ ${ }^{I}$ Physics Department, New York City College of Technology, \\ The City University of New York,Brooklyn, NY 11201, USA \\ ${ }^{2}$ The Graduate School and University Center, The City University of New York,New York, NY 10016, USA \\ e-mail: rkezerashvili@citytech.cuny.edu
}

\begin{abstract}
In this review, we try to summarize the results of experimental search and theoretical studies within variational methods, the method of Faddeev and Faddeev-Yakubovsky equations, and the method of hyperspherical harmonics for the lightest neutron and kaonic clusters. In particular, we discuss few-body neutron clusters: bineutron, trineutron and tetraneutron. Related to few-body kaonic clusters the results of calculations for binding energies and widths for the $K^{-} p p$ and $\bar{K} N N N$ kaonic systems are presented and discussed.
\end{abstract}

Key words: Trineutron, tetraneutron, kaonic clusters.

PACS: 21.10.Dr, 21.45.-v, 27.10.+h

\section{Introduction}

The cataclysmic events that occur near the end of the life of a star lead to one of only three possible final states: a white dwarf, a neutron star, or a black hole. The mass of the star, particularly that of the core, appears to be the primary factor in determining the final state. A more massive star would need to be hotter to balance its stronger gravitational attraction. While a star is burning, the heat in the star pushes out and balances the force of gravity. When the star's fuel is spent, and it stops burning, there is no heat left to counteract the force of gravity. How much mass the star had when it died determines what it becomes. Detailed calculations have shown that for star with mass less than about 1.4 times the mass of our sun electron degeneracy pressure permanently halts collapse. White dwarfs are stable cold stars that are supported by electron degeneracy pressure. Calculations show that stars that have between 1.4 and 3 times the mass of the sun implode into neutron stars that are the end product of stellar evolution, and their outer core is composed of neutrons at truly enormous densities. The central region of the neutron star is supported by the degeneracy pressure of neutrons. A star with mass greater than 3 times than of the sun gets crushed into a single point - a black hole.

At high density, when the sum of masses of a proton and electron and Fermi energy exceeds the neutron mass, it is energetically favorable to combine a proton and an electron into a neutron: $p+e^{-} \leftrightarrow n+$ $v_{e}$. Both neutron and neutrino rich matter are produced at the core. Therefore, at higher densities, matter becomes more and more neutron-rich. A progressive neutronization of matter at higher and higher densities makes a lower energy state. An attractive pairing interaction between neutrons, can couple them to form a state with integer spin and, therefore, paired neutrons act like bosons. These "bosons" can form a condensate-like state in which all of the bosons occupy the same quantum state and form a superfluid. Just as the pairing of protons that are charged fermions forms a superconductor. In that same general sense, we also can have superconductivity and superfluidity in neutron stars. Thus, we can have superconductivity and superfluidity in the outer core of neutron stars. Superconductivity and superfluidity, if observed in neutron stars, could tell us a lot about the pairing and hence inform us about aspects of nuclear physics that are mighty difficult to get from laboratories. Information on multineutron forces obtained in studies of multineutron systems is a critical input into theories of neutron stars $[1,2]$. Therefore, the study of dineutrons, trineutrons, tetraneutrons as well as multineutrons and neutron drops is important for understanding the structure and processes in neutron stars. From another side, early suggestions for kaon condensate in dense matter [3-6] motivated the search for bound states of kaons in nuclei, since the kaon-nucleus interaction could answer the question of whether kaon condensation takes place in the inner core of neutron stars. This is one of the reasons why the study of few-body kaonic systems have attracted much attention in the last decade. Today, our 
understanding of the details of the process of neutron star formation is not very well defined. This is presently an active area of research.

Below, I am reviewing and presenting the status of studies of three- and four-body neutron and kaonic systems.

\section{Few-body neutron clusters}

A simple fact has now been established: all nuclei that are heavier than the hydrogen nucleus are made up of both protons and neutrons. The question then arises as to whether a nuclei made up of only neutrons or protons can exist. On the basis of current knowledge the theoretical answer is probably ... Well, let's discuss this!

During the last 60 years, experimental search and theoretical investigation has continued to focus on atomic nuclei consisting only of neutrons. A recently reported observation of the tetraneutrons [25] and some theoretical results, however, revives old questions: do dineutrons, trineutrons and multineutrons nuclei exist? Can a nucleus be made up of neutrons only? Does neutron matter exist? The existence of a bound dineutron, multineutrons nuclei, neutron drops and neutron matter is of great importance, as it would challenge our understanding of nuclear few-body systems and the evolution of the universe. The answers to these questions would most certainly require a revision of modern realistic models of the nucleon-nucleon force and three-nucleon interaction and more over introduce a four-nucleon interaction.

A free neutron decays into a proton, an electron, and an antineutrino, which is associated with an electron. The time of this decay is about 1000 seconds. In other words, a free neutron may exist for only about 16 minutes. What about the existence of the system of two bound neutrons known as a dineutron? Searching resonances and bound states in a system of two neutrons is a well defined problem and numerically well under control. The two neutron resonances are associated with the poles of the $S$ -matrix, which are embedded in the fourth-quadrant of the complex $k$ plane. They are solutions of the time-dependent Schrödinger equation without the incoming wave and the outgoing wave increasing exponentially at infinity. In Ref. [7] it was mentioned that nonrealistic Volkov potentials [8] do have bound dineutrons. However, these potentials are not realistic; they produce bound ${ }^{2} n$, with the same binding energies as their deuterons; they have no tensor or $L S$ terms; and they cannot reproduce modern phase shift analyses in any partial wave [7]. Theoretical calculations show no existence of the resonance or bound states in the system of two neutrons for all the existing realistic and phenomenological models of the nucleon-nucleon interaction. Experimental searches of the dineutron have been also performed using different nuclear reactions but no evidence for the existence of the ${ }^{2} n$ has been found. Thus, today we are confident that dineutrons do not exist but could very nearly exist: a slight increase in the attraction between the two particles would result in a bound structure, the dineutron being formed. However, in neutron-rich matter like a neutron star where the density 3-times as much as the normal nuclear density would nucleon-nucleon interaction modified so that brings two neutrons to be bound. This question needs to be addressed. Research into the possibility that nuclei have more than two neutrons shows that, very often, adding a further neutron increases the stability of the structure. The question then arises as to whether a neutron system made up of more than two neutrons could exist.

A three-neutron resonance has not yet been firmly established. The weight of early experimental evidence reviewed in Ref. [9] is strongly against the existence of a bound state of the three-neutron system, and only controversial evidence of a three-neutron resonance was cited. The situation up to 1987 has been reviewed in the compilation [10]. Reference [11] reported the possible existence of the trineutron through the reaction ${ }^{3} \mathrm{H}(n, p)^{3} \mathrm{n}$. Later, the same reaction was studied in Ref. [12] and no evidence for the existence of the trineutron was found. Searches for a bound state of the three neutron were conducted in reactions: ${ }^{3} \mathrm{H}\left(\pi^{-}, \gamma\right)^{3} \mathrm{n},{ }^{3} \mathrm{H}\left(\pi^{-}, \pi^{+}\right)^{3} \mathrm{n}$ and ion collision reactions such as ${ }^{7} \mathrm{Li}\left({ }^{11} \mathrm{~B},{ }^{15} \mathrm{O}\right)^{3} \mathrm{n}$ and ${ }^{2} \mathrm{H}\left({ }^{12} \mathrm{C},{ }^{13} \mathrm{~N}\right){ }^{3} \mathrm{n}$. As of yet, none of these reactions have provided evidence for a bound trineutron. The most intensive search for the prediction of a bound trineutron has been performed using a pionic double charge exchange reaction ${ }^{3} \mathrm{He}\left(\pi^{-}, \pi^{+}\right)^{3}$ n. An investigation [13] of the process ${ }^{3} \mathrm{He}\left(\pi^{-}, \pi^{+}\right)^{3} \mathrm{n}$ found no evidence of the existence of the ${ }^{3} n$ or resonance state of three neutrons. Earlier study [14] pointed to resonance in the three neutrons. However, the resonance behavior can be explained by the final state interaction of three neutrons in continuum spectrum as was demonstrated in Refs. $[15,16]$. The double charge exchange process on ${ }^{3} \mathrm{He}$ was also investigated in Ref. [17], which while criticizing previous work [14] pointed again to a three-neutron resonance around $12 \mathrm{MeV}$ excitation. For a 
trineutron the bound state has been studied extensively in the last four decades resulting in a numerically precise solution of the Faddeev equations in momentum and coordinate space, and using the hyperspherical functions method. Independent of the theoretical framework, such as the Faddeev formalism [18, 19], the method of the hyperspherical functions or variational calculations, most theoretical works do not predict a bound ${ }^{3} \mathrm{n}$ state in the three-neutron system. However, it has been stressed in Ref. [20] that subtle changes in the nucleon-nucleon potential, which would not affect results from phase shift analyses, may lead to bound neutronic nuclei. To summarize, although the double charge exchange reaction of negative pions on ${ }^{3} \mathrm{He}$ nucleus has been examined at various incident energies of pion, from the analysis of the invariant mass spectra for three neutrons, no evidence for the bound trineutron has been found. However, a calculation [21] predicts a resonance state with the width of $13 \mathrm{MeV}$ in the three-neutron system. Although such a resonance would easily fit early interpretation of data on pionic double charge exchange on ${ }^{3} \mathrm{He}$ [14] (this observation was supported by measurements reported in Ref. [17] in 1986), more recent investigations $[13,22,23]$ of this process do not give any experimental evidence for it.

Several more recent experiments have strengthened the evidence against the bound trineutron and have failed to discover a resonance structure that cannot be otherwise explained. The study [24] shows that realistic nucleon-nucleon interaction models exclude any possible experimental signature of three-neutron resonances. Thus, today there is no unambiguous answer for the existence of the three-neutron nucleus. Apart from these aspects the question of whether multineutron systems exist is of principal interest by itself.

This year, a candidate resonant tetraneutron state with the energy of $0.83 \pm 0.65$ (stat) \pm 1.25 (syst) $\mathrm{MeV}$ above the threshold of four-neutron decay has been found in the missing-mass spectrum obtained in the double-charge-exchange reaction ${ }^{4} \mathrm{He}\left({ }^{8} \mathrm{He},{ }^{8} \mathrm{Be}\right)$ at $186 \mathrm{MeV} / \mathrm{u}$ [25]. The experiment was performed at the RI Beam Factory at RIKEN. Previously, in experiments, the system of four bound neutrons ${ }^{4} n$ was searched through using heavy-ion transfer reactions such as ${ }^{7} \mathrm{Li}\left({ }^{11} \mathrm{~B},{ }^{14} \mathrm{O}\right){ }^{4} \mathrm{n}[26],{ }^{7} \mathrm{Li}\left({ }^{7} \mathrm{Li},{ }^{10} \mathrm{C}\right)^{4} \mathrm{n}$ [27], and the pion double charge exchange reaction ${ }^{4} \mathrm{He}\left(\pi^{-}, \pi^{+}\right)^{4}$ n. Early measurements of the ${ }^{4} \mathrm{He}\left(\pi^{-}\right.$, $\left.\pi^{+}\right)^{4} n$ reaction carried out in search of evidence for ${ }^{4} n$ are summarized in the compilation [28]. No bound ${ }^{4} n$ was detected in these early works. Later the momentum spectrum from the pion double charge exchange reaction was measured in Ref. [29] in a search for ${ }^{4} \mathrm{n}$. Note, however, that the theoretical study of Ref. [30] reported that the final-state interaction in the four-neutron system in continuum spectrum is so strong that the tetraneutron could not be observed in the kinematic region explored in Ref. [29]. Pion spectra and total cross sections for pion double charge exchange were also measured in Refs. [31 - 33] for different incident pion energies. No evidence for ${ }^{4} \mathrm{n}$ was obtained. Several attempts have been made to find a bound tetraneutron system by using a uranium fission reaction $[34,35,36]$ and the experimental observation of ${ }^{4} \mathrm{n}$ was claimed in the interaction of $100 \mathrm{MeV} \alpha$-particle with uranium nucleus in Ref. [36].

Several theoretical studies of pion double charge exchange on ${ }^{4} \mathrm{He}$ have been reported. In Ref. [37] cross sections were calculated in a model in which two single charge exchange scatterings occur. The reaction was studied in the framework of a four-body hyperspherical basis method in Ref. [38] but existing experimental data were interpreted without bound or resonance state of four neutrons. No bound tetraneutron was found in Ref. [39] within the angular potential functions method, in Ref. [40] using the stochastic variational method and in Refs. [41] and [42] within the hyperspherical functions method. In contrast, calculations within the hyperspherical functions method led the authors in Ref. [43] to the conclusion that the tetraneutron may exist as a resonance only for the $N N$ potential that binds the dineutron.

In the new millennium, an experimental search and theoretical study of tetraneutron transitioned to a new phase. In 2002 an international team led by physicists from the Particle Physics Laboratory of Caen, have presented in Ref. [44] experimental results suggesting the existence of a bound tetraneutron. These results have been obtained by using the exotic beams of the French national large heavy-ion accelerator in Caen and by studying the breakup reaction of ${ }^{14} \mathrm{Be}$ into ${ }^{10} \mathrm{Be}$ and bound tetraneutron. The heavy-ion transfer reactions, and the pion double charge exchange reaction require considerable reconfiguration of the target nuclei and should be strongly suppressed. In contrast, the nucleus ${ }^{14} \mathrm{Be}$ consists of a strongly bound core ${ }^{10} \mathrm{Be}$ and four weakly bound neutrons that could form a tetraneutron-like configuration, which might be shaken off in the ${ }^{14} \mathrm{Be}$ breakup. In experiment [44] events were observed that exhibit the tetraneutron cluster liberated in the breakup of ${ }^{14} \mathrm{Be}$. The lifetime suggested by this measurement would indicate that 
the tetraneutron is a stable particle. In 2016, the resonant tetraneutron state was found in the reaction ${ }^{4} \mathrm{He}\left({ }^{8} \mathrm{He},{ }^{8} \mathrm{Be}\right)$ [25]. If confirmed, these discoveries, which would call into question current theoretical models, will have major repercussions in the field of nuclear physics.

The existence of the bound tetraneutron system was also discussed in theoretical studies [45 - 49]. In Ref. [46] it was proposed that, if tetraneutron existed, it could be formed by a bound state of two dineutron molecules. The possibility for a tetraneutron to exist as a low-energy resonance state was studied in Ref. [49]. In [45] the hyperspherical functions method and realistic nucleon-nucleon interactions have been used to argue against the existence of a tetraneutron. It was pointed out that due to the small probability for a pair of neutrons to be in the singlet even state, the two-body nuclear force cannot by itself bind four neutrons, even if it could bind a dineutron. An unrealistic modification of the nucleon-nucleon force or introduction of unrealistic four-nucleon forces would be needed to bind a tetraneutron. As for experimental searches of the other light bound multineutron systems, the calculations presented in Ref. [45] suggest that they might be unsuccessful. It is also important to mention that Ref. [7] shows that it does not seem possible to change any modern twoand three-nucleon interaction to bind a tetraneutron without destroying many other successful predictions of these interactions. This means that, should recent experimental claims $[44,25]$ of a bound tetraneutron be confirmed, our understanding of nuclear forces will have to be significantly changed.

Theoretical investigations of multineutrons have been carried out for a system of six, eight and ten bound neutrons using the hyperspherical functions method with different nucleon-nucleon interactions [45]. Results of calculations show no bound states for these neutron systems. Theorists are also studying neutron drops [50, 51]. Neutron drops are collections of neutrons held together by both an external nuclear well and the interaction between neutrons. The properties of these drops can be used as "data" for fitting simpler effective interaction models that are employed in the study of large neutron-rich nuclei, the crusts of neutron stars, and neutron matter.

\section{Few-body kaonic clusters}

Kaonic nuclei carry important information concerning the $\bar{K}$ - nucleon interaction in the nuclear medium. This information is very important in understanding kaon properties at finite density and in determining constraints on kaon condensation in high-density matter. The latter will allow one to adjust the methods developed in condensed matter physics for exciton and excitonic polariton condensates (see, for example, $[52,53]$ ) to study the kaon condensation. The best way to understand the many body kaonic nuclear system is to study the simplest three- and four-body clusters: $\bar{K} N N$, and $\bar{K} N N N$. The light kaonic clusters $\bar{K} N N$, and $\bar{K} N N N$ represent three- and four-body systems and theoretically can be treated within the framework of few-body physics approaches. In the recent past much efforts have been focused on the calculations of quasibound state energies and widths for threeand four-body kaonic clusters. A variety of methods have been used in configuration and momentum spaces, to obtain eigenvalues for energy and width of quasibound states using diverse sets of $\bar{K} N$, and $N N$ interactions. These include but are not limited by variational method approaches [54-65], the method of Faddeev equations in momentum and configuration spaces $\left[\begin{array}{lll}67 & - & 79\end{array}\right]$, Faddeev-Yakubovsky equations [77] and the method of hyperspherical harmonics in configuration and momentum spaces $[80,81,79]$.

On the experimental side, several experiments have been performed to search for the kaonic clusters using various nuclear reactions starting from the first measurement reported by the FINUDA collaboration for the $K^{-} p p$ cluster [82] and including the most recent reports of J-PARC E15 and J-PARC E27 collaborations [83, 84] and HADES collaboration [85]. Recent HADES collaboration partial wave analysis of the reaction $p p \rightarrow p K^{+} \Lambda$ at $3.5 \mathrm{GeV}$ to search for the $K^{-} p p$ bound state shows that at a confidence level $95 \%$ such a cluster cannot contribute more than $2-12 \%$ to the total cross section with a $p K^{+} \Lambda$ final state [85]. However, there are important reports of $K^{-} p p$ experimental searches done by the DISTO and J-PARC E27 collaborations. They reported some signal at $100 \mathrm{MeV}$ below the $K^{-}$ and two protons threshold, which may be related to the kaonic cluster $K^{-} p p$. J-PARC E27 collaboration has observed a $K^{-} p p$-like structure in the $d\left(\pi^{+}, K^{+}\right)$ reaction at $1.69 \mathrm{GeV} / \mathrm{c}$, while Ref. [86] reports an indication of a deeply bound $K^{-} p p$ state in the $p p \rightarrow$ $p \Lambda K^{+}$reaction at $2.85 \mathrm{GeV}$. The authors of Refs. [87], [88] (experiment E471) announced the experimental discovery of the bound state $\bar{K} p p n$, named $S^{0}(3115)$, with quantum numbers $I\left(J^{P}\right)=1\left(\frac{3^{+}}{2}\right)$. The results have been checked in a new experiment 
with larger statistics, and have been withdrawn. The ambiguous situation with the search of kaonic clusters has led the KEK-PS collaboration, experiment E549 [89], [90] to carry out a new experimental search with improved resolution and higher statistics compared with the E471 experimental setup. In the search for the neutral tribaryon with strangeness $S=-1$ and isospin 1 by missing-mass analysis of the inclusive ${ }^{4} \mathrm{He}\left(K_{\text {stopped }}\right.$, $p$ ) reaction with the quite high statistics for protons, no statistically significant signal of the narrow structure was observed. Therefore, the situation is still controversial and the existence, for example, of the $K^{-} p p$ quasibound state has not been established yet. Thus, the theoretical and experimental study of composite systems of $K-$ mesons and nucleons is still a challenging issue in nuclear physics.

Table 1 - Summary of the theoretical studies for the $K^{-} p p$ cluster.

\begin{tabular}{|c|c|c|c|c|}
\hline Method & $\mathrm{B}\left(K^{-} p p\right) \mathrm{MeV}$ & Width, $\Gamma \mathrm{MeV}$ & $\bar{K} N$ & References \\
\hline Variational & 48 & 61 & AY & {$[54],[55],[59]$} \\
\hline \multirow[t]{4}{*}{ Methods } & $20 \pm 3$ & $40-70$ & Chiral model & {$[62],[63]$} \\
\hline & $40-80$ & $40-85$ & Sep. & {$[64]$} \\
\hline & $20-35$ & $20-65$ & Chiral model & {$[65]$} \\
\hline & 124 & 12 & AY & {$[66]$} \\
\hline Methods of & $47-70$ & $90-100$ & Sep. En. Indep. & [67], [68], [78] \\
\hline Faddeev & $\sim 32$ & $\sim 50-65$ & Sep. En. Dep. & {$[78]$} \\
\hline \multirow[t]{6}{*}{ equations } & $45-95$ & $45-80$ & Sep. En. Indep. & {$[69],[70],[72]$} \\
\hline & $9-16$ & $34-40$ & Sep. En. Dep. & {$[72]$} \\
\hline & $30-40$ & $50-80$ & & [73]-[76] \\
\hline & $\sim 52$ & & Sep. En. Indep. & {$[77]$} \\
\hline & $46.3-47.3$ & & AY & [79] \\
\hline & $20.6-21.6$ & & HW & [79] \\
\hline \multirow[t]{5}{*}{ Methods of $\mathrm{HH}$} & $\sim 16$ & $\sim 41$ & Chiral model & {$[80]$} \\
\hline & $15-17$ & $36-43$ & Chiral model & {$[81]$} \\
\hline & $40-48$ & $75-96$ & AY & {$[81]$} \\
\hline & $46.3-46.5$ & $74.5-84.3$ & AY & [79] \\
\hline & $20.5-206$ & $48.1-49.5$ & HW & [79] \\
\hline
\end{tabular}

Let us focus on results of the calculations of the binding energy and width for the three-body $\bar{K} N N$ and four-body $\bar{K} N N N$ kaonic clusters. To describe these system were used an energy-independent and energy-dependent local as well as a separable $\bar{K} N$, effective interactions. The local energy-independent effective $\bar{K} N$, interaction was constructed in Ref. $[54,59]$ based on a phenomenological approach so as to reproduce the existing experimental data for the $\bar{K} N$ scattering length, the mass and width of the $\Lambda$ (1405) hyperon and the $1 s$ level shift caused by the strong $\bar{K} N$, interaction in the kaonic hydrogen atom. We refer to this potential as the Akaishi-Yamazaki (AY) potential. The energy-dependent local effective $\bar{K} N$, interaction given in Ref. [91] was derived based on the chiral unitary approach for the $s$ - wave scattering amplitude with strangeness $S=-1$, and reproduces the total cross sections for the elastic and inelastic $K^{-} p$ scattering, threshold branching ratios, and the $\pi \Sigma$ mass spectrum associated with the $\Lambda$ (1405). Hereafter we refer to this potential as the HW potential. For the calculations of the binding energy and the width with the variational method, the method of hyperspherical harmonics $(\mathrm{HH})$ and the Faddeev equations in configuration space as input for the $\bar{K} N$, interaction were used the energy-dependent effective HW and the phenomenological AY potentials, and different $N N$ 
interaction. When the kaonic clusters treated within the Faddeev or Faddeev-Yakubovsky equations separable potentials are used as the input for the $N N$ and $\bar{K} N$, interactions.

Calculations for a binding energy and width of the kaonic three-body system are presented in Table 1 are performed using different potentials for the $N N$ interaction, as well as the energy-independent and the energy-dependent effective potentials for the description of the kaon-nucleon interaction. Such an approach allowed us to examine how the $K^{-} p p$ cluster's structure depends on different choices of the $\bar{K} N$, interactions for the same $N N$ potential, as well as to investigate its dependence on different choices of the $N N$ interaction for the same $\bar{K} N$, interaction, and to understand the sensitivity of the system to the input interactions. Therefore, the use of different $N N$ potentials and $\bar{K} N$, interactions allows one to perform a validity test for the lightest kaonic clusters against various $N N$ and $\bar{K} N$, interactions.

One can address the theoretical discrepancies in the binding energy and the width for the $K^{\prime} p p$ system presented in Table 1 related to the different $N N$ and $\bar{K} N$, interactions or a method of calculations. However, analysis of theoretical studies indicates that mostly the discrepancies of the results of calculations for the binding energy and the width are related to the treatment of effective $\bar{K} N$, interaction. The binding energy found in Ref. [79] using the differential Faddeev equations and $\mathrm{HH}$ methods are in good agreement with the one obtained with the variational method. This is a good sign that the binding energy does not depend significantly on the method of calculation. Different variational approaches are of comparable quality in their high degree of consistency and all results are consistent. Differences are mostly due to a different $\bar{K} N$, input and possibly slightly due to the $N N$ input.Variational calculations as well as calculations using the differential Faddeev equations and $\mathrm{HH}$ methods confirm that the effective $\bar{K} N$, interaction derived from chiral $\mathrm{SU}(3)$ dynamics yields a shallowly bound $K^{-} p p$ cluster, while the phenomenological energy independent AY potential predicts much deeper binding energy for all considered $N N$ interactions. Most importantly, the results support the conclusion that the key role in binding the $K^{-} p p$ system is played by the $\bar{K} N$ interaction and the $\bar{K} N$ potential obtained based on chiral SU(3) dynamics leads to binding energies of relatively low values.
The calculations with the Faddeev equations for the three-body system with coupled $\bar{K} N N$ and $\pi \Sigma N$ channels performed in Refs. [67], [68] with separable two-body potentials yield larger bindings than obtained in a similar approach in Ref. [69]. Later, two of the authors of [69] repeated their calculation in $[70,72]$ using two models with the energy-independent and energy-dependent potentials for the $s$ - wave $\bar{K} N$, interaction, and their calculations yield smaller values for the binding energy 44-58 MeV and width 34-40 MeV [72]. The Faddeev calculations [78] for the $\bar{K} N N$ quasibound state with the two phenomenological and the energy-dependent chirally motivated models of the $\bar{K} N$ interaction lead to the following results for the K pp cluster: $32 \mathrm{MeV}$ with the chirally motivated models and $47-54 \mathrm{MeV}$ with the phenomenological $\bar{K} N$ potentials. Therefore, one can conclude that the Faddeev calculations for the energy-independent models for the $\bar{K} N$ interaction predict a deeper binding energy than that of the energy-dependent description of the $\bar{K} N$ interaction.

Recently, Faddeev-Yakubovsky calculations [77] were made for the four-particle $K^{-} p p$ kaonic cluster, where the quasibound states were treated as bound states by employing real $s$ - wave two-body separable potential models for the $\bar{K}$-nucleon interactions as well as for the $N N$ interaction. Fully four-body nonrelativistic realistic calculations of $\bar{K} N N N$, quasibound states within the method of $\mathrm{HH}$ in configuration space, using realistic $N N$ potentials and subthreshold energy dependent chiral $\bar{K} N$ interactions, were presented in Ref. [80]. Given that below we present the results of our calculations for the $\bar{K} N N N$ quasibound states in the framework of the method of $\mathrm{HH}$ in momentum representation using AV18 [93] and M [96] $N N$ potentials and AY and HW $\bar{K} N$, interactions as inputs. To find the binding energies with the above-mentioned set of potentials, we solve a system of coupled integral equations for the hyperradial functions [79]. In the calculations we limit our consideration with the value 10 for the global momentum getting a reasonable convergence for the binding energy. In Table 2 we present our results for the $\bar{K} N N N$, cluster that we compare with those obtained via different methods. The results of our calculations for the energy and the width show dependence on the $N N$ potentials and on the $\bar{K} N$, interactions. However, this dependence is dramatically different: for the same $\bar{K} N$ interaction 
and different $N N$ potentials the ground state energy and the width change by about $3-15 \%$, while for the same $N N$ potential and different $\bar{K} N$ interaction the energy changes by a factor of more than 3 and the width changes by more than twice. The comparison of our results for the $K^{-} p p n$ and $K^{-} p n n$ clusters obtained for theAV18 $N N$ interaction and the HW $\bar{K} N$ interaction with calculations [80] within the variational $\mathrm{HH}$ method for the AV14 $N N$ interaction and shallow chiral $\bar{K} N$ interaction shows a reasonable agreement. The predictions [58] for the binding energy and the width for the kaonic clusters studied based on a framework of antisymmetrized molecular dynamics and employing adopted AY [54] potential as a bare $\bar{K} N$ interaction are presented in the last column of Table 2. As is seen from Table 2 there is a reasonable agreement for the widths for the $K^{-} p p n$ and $K^{-} p n n$ clusters among the present study and study [80] in case of HW potential. However, the widths of the $K^{-} p p n$ and $K^{-} p p p$ systems are largely different among our study and an earlier study [58] in the case of the AY potential. Interestingly enough, our calculations for the AY interaction indicate that for the system $\bar{K} N N N$, the cluster $K^{-} p p p$ is more deeply bound than the Kppn, which contradicts the results [58]. In a shell-model picture, one of three protons in the $K p p p$ should be raised up to $0 p$ orbit due to the Pauli principle, while all nucleons in $K^{-} p p n$ occupy the $0 s$ orbit. So, the nave expectation is that the $K^{-} p p n$ is energetically lower than the $K^{-} p p p$. From the other side the larger number of the strongly attractive $K^{-} p$ pairs in the $\left(\frac{3}{2}^{+}, 1\right)$ state than in the $\left(\frac{1}{2}^{-}, 0\right)$ state may cause a lowering of the $T=1$ state, even below the $T=$ 0 , although the third proton in the $T=1$ state should be flipped up to the orbital $\left(0 p_{3 / 2}\right)$. The final picture depends on the strength of the $\bar{K} N$ interaction. The attractive AY interaction is much stronger than the effective HW interaction. The strength of the $\bar{K} N$ interaction plays an important role that may lead to the nuclear compression.

Table 2 - The binding energy $B$ and width $\Gamma$ for the $\bar{K} N N N$ system calculated in the framework of the method of HH in the momentum representation for different interactions with results from Refs. [58], [77] and [80]. The parity $\pi$ includes the eigen parity of antikaon

\begin{tabular}{|c|c|c|c|c|c|c|c|c|c|c|}
\hline & $J^{\pi}$ & $T$ & & \multicolumn{2}{|c|}{ AV18 } & \multicolumn{2}{|c|}{ M } & {$[77]$} & {$[80]$} & {$[58]$} \\
\hline & & & & AY & HW & AY & & HW & & \\
\hline$K^{-} p p n$ & $\frac{1^{-}}{2}$ & 0 & $B, \mathrm{MeV}$ & 92.1 & 28.6 & 97.9 & 29.3 & 28.9 & 69 & 110.3 \\
\hline & & & $\Gamma, \mathrm{MeV}$ & 83.4 & 30.3 & 84.1 & 32.9 & 30.8 & & 21.2 \\
\hline$K^{-} p n n$ & $\frac{1^{-}}{2}$ & 1 & $B, \mathrm{MeV}$ & 64.6 & 17.2 & 66.7 & 18.5 & 18.7 & & \\
\hline & & & $\Gamma, \mathrm{MeV}$ & 74.2 & 27.1 & 80.4 & 31.0 & 31.4 & & \\
\hline$K^{-}$ppp & $\frac{3^{+}}{2}$ & 1 & $B, \mathrm{MeV}$ & 101.9 & 25.8 & 107.6 & & 28.1 & & 96.7 \\
\hline & & & $\Gamma, \mathrm{MeV}$ & 87.9 & 28.1 & 89.8 & & 31.2 & & 12.5 \\
\hline
\end{tabular}

\section{Conclusions}

Today there is no unambiguous answer for the existence of the trineutron as a bound or resonance state. There are three claims of the experimental observation of ${ }^{4} \mathrm{n}$ : one in a fission reaction [36], and the recent two in the breakup reaction of ${ }^{14} \mathrm{Be}$ into ${ }^{10} \mathrm{Be}$ and ${ }^{4} \mathrm{n}$ [44], and in the double-charge-exchange reaction ${ }^{4} \mathrm{He}\left({ }^{8} \mathrm{He},{ }^{8} \mathrm{Be}\right)$ [25]. However, in theoretical studies, no evidence for ${ }^{4} \mathrm{n}$ was obtained within the existing modern two- and three-nucleon interaction. If the experimental discoveries [44] and [25] would be confirmed, this would call into question current theoretical models of nuclear forces.

The results of calculations within the different theoretical methods show that the binding energy and the width of the $K^{-} p p$ system depend entirely on the ansatz for the $\bar{K} N$ interaction and substantially change when the AY and HW potentials are used. The predicted values for the binding energy and the 
width are in considerable disagreement.Model calculations with the $\bar{K} N$ interaction derived based on the chiral unitary approach predict a shallow binding state with large width for the $K^{-} p p$ cluster. For example, for the $K^{-} p p$ cluster, the predicted values for the binding energy and the width are 9-95 $\mathrm{MeV}$ and $20-110 \mathrm{MeV}$, respectively. The sensitivity of the binding energy and the width to the details of the $N N$ potentials as long as the $K^{-} p p$ kaonic system is strongly or weakly bound dependence on different types ofinteraction is weak.
Finally, the situation is still controversial and the existence of theandquasibound states have not been established yet. Many calculations show that, for theand four-particle kaonic clusters, the binding energy is larger or comparable to the width. As a consequence, perhaps, we are facing a situation in which it is hard to identify the resonances that would make the experimental observation challenging. Thus, the theoretical and experimental study of composite systems of mesons and nucleons is still a challenging issue in nuclear physics.

\section{References}

[1] P. B. Demorest. T. Pennucci. S.M. Ransom. M.S.E. Roberts. and J.W.T. Hessels. Nature (London)2010. - Vol. 467.- P. 1081. P. 787 .

[2] E. Hiyama. Few-Body Syst. - 2015. - Vol. 56.-

[3] D. B. Kaplan and A. E. Nelson. Strange goings on in dense nucleonic matter // Phys. Lett. B. - 1986. Vol. 175. - P. 57.

[4] G. E. Brown. The equation of state of dense matter: supernovae. neutron stars and black holes // Nucl. Phys. A. - 1994. - Vol. 574. - P. 217.

[5] G. E. Brown. M. Rho. Chiral restoration in hot and/or dense matter // Phys. Rep. - 1996. - Vol. 269. P.333.

[6] C. H. Lee. Kaon condensation in dense stellar matter// Phys. Rep. - 1996. - Vol. 275. - P. 255.

[7] S. C. Pieper. Can Modern Nuclear Hamiltonians Tolerate a Bound Tetraneutron? // Phys. Rev. Lett. - 2003. - Vol. 90. - P. 252501.

[8] A. B. Volkov. Equilibrium deformation calculations of the ground state energies of $1 \mathrm{p}$ shell nuclei // Nucl. Phys. - 1965. - Vol. 74. - P. 33.

[9] S. Fiarman. S. S. Hanna. Energy levels of light nuclei $A=3 / /$ Nucl. Phys. A. - 1975. - Vol. 251. - P. 1.

[10] D. R. Tilley. H. R. Weller. and Hasan. Energy levels of light nuclei $\mathrm{A}=3$ // Nucl. Phys. A. - 1987. Vol.474. - P. 1.

[11] V. Ajdaic. M. Cerineo. B. Lalovi. G. Pai. I. laus. and P. Toma. Reactions H3(n.p) $3 n$ and H3(n.H4) $\gamma$ at En=14.4 MeV // Phys. Rev. Lett. - 1965. - Vol. 14. P.444.

[12] S. T. Thornton. J.K. Bair. C.M. Jones. and H.B. Willard. Search for the Trineutron // Phys. Rev. Lett. 1966. - Vol. 17. - P. 701.

[13] J. Grater. P. A. Amaudruz. R. Bilger. et al. // Eur. Phys. J. A. - 1999. - Vol. 4. - P. 5

[14] J. Sperrinde. et al. // Phys. Lett. B. - 1970. Vol.32. - P. 185 .

[15] R. I. Jibuti. R. Ya. Kezerashvili. Sov. J. Nucl. Phys. - 1984. - Vol. 39. - P. 264.

[16] R.I. Jibuti. R.Ya. Kezerashvili. Double-charge-ex change reactions of $\pi$-mesons on three- and four-particle nuclei // Nucl. Phys. A. - 1985. - Vol.437. - 1q 687.
[17] A Stez et al.Pion double charge exchange on $3 \mathrm{He}$ and 4He // Nucl. Phys. A. - 1986. - Vol. 457. - P. 669.

[18] H. Witala. W. Glöckle. Resonances in the three-neutron system // Phys. Rev. C. - 1999. - Vol. 60. P. 024002.

[19] A. Hemmdan. W. Glöckle. and H. Kamada. Indications for the nonexistence of three-neutron resonances near the physical region // Phys. Rev. C. 2002. - Vol. 66. - P. 054001.

[20] A. I. Baz. V.N. Bragin. Do multineutrons really exist? // Phys. Lett. B. - 1972. - Vol. 39. - P. 599.

[21] A. Csoto. H. Oberhummer. R. Pichler. Searching for three-nucleon resonances // Phys. Rev. C. - 1996. Vol. 53. - P. 1589

[22] J. Grater. et al.Inclusive measurements of pionic double charge exchange on $3 \mathrm{He}$ at low energies // Phys. Lett. B. - 1999. - Vol. 471. - P. 113.

[23] M. Yuly et al.Pion double charge exchange and inelastic scattering on 3He // Phys. Rev. C. - 1997. Vol.55. - P. 1848.

[24] R. Lazauskas and J. Carbonell. Three-neutron resonance trajectories for realistic interaction models // Phys. Rev. C. - 2005. - Vol. 71. - P. 044004.

[25] K. Kisamori. et al. Candidate Resonant Tetraneutron State Populated by the He4(He8,Be8) Reaction// Phys Rev. Lett. - 2016. - Vol. 116. -P.052501.

[26] A. V. Belozyorov. C. Borcea. Z. Dlouhy. and A. M. Kalininю Search for the tri- and tetra-neutron in reactions induced by $11 \mathrm{~B}$ and $9 \mathrm{Be}$ ions on $7 \mathrm{Li} / / \mathrm{Nucl}$. Phys. A. - 1988. - Vol. 477. - P. 131. P. 3.

[27] D. V. Aleksandrov. et al. Yad. Fiz. 47. - 1988. -

[28] D. V. Aleksandrov. E. Y. Nikol. B. G. Novatski. S. B. Sakuta. and D. N. Stepanov.Search resonances in systems of three and four neutrons in the reactions $7 \mathrm{Li}$ (7Li, 11C) and 7Li (7Li, 10C) in the nuclei 7Li // JETP Lett. - 2005. - Vol. 81. - P. 43.

[29] S. Fiarman. W.E. Meyerhof. Energy levels of light nuclei $\mathrm{A}=4$ // Nucl. Phys. A. - 1973. - Vol. 206. P.1.

[30] J. E. Urgan. et. al.Search for the tetraneutron by the double-charge-exchange of negative pions // Phys. Lett. B. - 1984. - Vol. 144. - P. 333. 
[31] R. Ya. Kezerashvili. Sov. J. Nucl. Phys. - 1986. Vol. 44. - P. 542.

[32] A. Stetz. at. al.Pion Double Charge Exchange on He4 and Meson Exchange Currents// Phys. Rev. Lett. 1981. - Vol. 47. - P. 782

[33] E. R. Kinney. et. al.Inclusive pion double charge exchange in He4// Phys. Rev. Lett. - 1986. - Vol. 57. P.3152.

[34] T. P. Gorringe. et. al.Search for the tetraneutron using the reaction $\mathrm{He} 4(\pi-, \pi+) 4 \mathrm{n} / /$ Phys. Rev. C. -1989 . Vol. 40. - P. 2390.

[35] J. P. Schiffer and R. Vanderbosch. Search for a particle-stable tetra neutron // Phys. Lett. 5. - 1963. P.292.

[36] S. Cierjacks. G. Markus.W. Michaelis. and W. Ponitz // Phys. Rev. B. - 1965. - Vol. 345. - P. 137.

[37] V. A. Ageev. I. N. Vishnevskii. V. I. Gavrilyuk. et.al. Ukr. J. Phys. - 1986. - Vol. 31. - P. 1771.

[38] W. R. Gibbs. B. F. Gibson. A. T. Hess. and G. J. Stephenson. Pion double charge exchange on He4 // Phys. Rev. C. - 1977. - Vol. 15. - P. 1384.

[39] R. I. Jibuti. R. Ya. Kezerashvili. K. I. Sigua. Investigation of $\pi^{-}\left(\pi^{+}\right)+4 \mathrm{He} \rightarrow \pi+\left(\pi^{-}\right)+4 \mathrm{n}(4 \mathrm{p}) / /$ Phys. Lett. B. - 1981. - Vol. 102. - P. 381;

[40] Yad. Fiz. - 1980. - Vol. 32. - P. 1536

[41] A. M. Gorbatov. et. al. Yad. Fiz. - 1989. Vol.50. - P. 347.

[42] K. Vagra. Y. Suzuki.Precise solution of few-body problems with the stochastic variational method on a correlated Gaussian basis // Phys. Rev. C. - 1995. Vol.52. - P. 2885.

[43] A. M. Badalyan. T. I. Belova. N. B. Konyuhova. V.D. Efros. Sov. J. Nucl. Phys. - 1985. - Vol. 41. - P.926.

[44] S. A. Sofianos S. A. Rakityansky. G. P. Vermaak. Subthreshold resonances in few-neutron systems // J.Phys. G: Nucl. Part. Phys. - 1997. - Vol. 23 - P. 1619.

[45] I. F. Gutich. A. V. Nesterov. I. P. Okhrimenko Yad. Fiz. - 1989. - Vol. 50. - P. 19.

[46] F. M. Marques. et. al.Detection of neutron clusters// Phys. Rev. C. - 2002. - Vol. 65. - P. 0444006.

[47] N. K. Timofeyuk.Do multineutrons exist? // J.Phys. G: Nucl. Part. Phys. - 2003. - Vol. 29. - P. L9 .

[48] C. A. Bertulani and V. Zelevinsky.Is the tetraneutron a bound dineutron-dineutron molecule? // J.Phys. G. - 2003. - Vol. 29. - P. 2431.

[49] L.V. Grigorenko. N. K. Timofeyuk. and M.V.Zhukov. Broad states beyond the neutron drip line // Eur. Phys. J. A. - 2004. - Vol. 19. - P. 187.

[50] I. V. Simenog. B. E. Grinyuk. Yu. M. Bidasyuk. Asymptotic properties of structuralfunctions of nucleus6Li and 6He whitin three-particle models // Ukr. J. Phys. - 2006. - Vol. 51. - P. 954.

[51] Y. A. Lashko and G. F. Filippov. Cluster Structure of a Low-Energy Resonance in Tetraneutron // Phys. At. Nucl. - 2008. - Vol. 71. - P. 209.

[52] B. S. Pudliner. A. Smerzi. J. Carlson. V. R. Pandharipande. S. C. Pieper. and D. G. Ravenhall // Phys. Rev. Lett. - 1996. - Vol. 76. - P. 2416.

[53] P. Maris. J. P. Vary. S. Gandolfi. J. Carlson. and S. C. Pieper. Properties of trapped neutrons interacting with realistic nuclear Hamiltonians // Phys. Rev. C. 2013. - Vol. 87. - P. 054318.

[54] O.L. Berman. R.Ya. Kezerashvili. G.V.Kolmakov. and Yu.E. Lozovik. Turbulence in a Bose-Einstein condensate of dipolar excitons in coupled quantum wells // Phys. Rev. B. - 2012. - Vol. 86. - P.045108.

[55] O. L. Berman. R. Ya. Kezerashvili. K. Ziegler // Phys. Rev. B. - 2012. - Vol. 86. - P. 235404.

[56] Y. Akaishi and T. Yamazaki. Superfluidity and collective properties of excitonic polaritons in gapped graphene in a microcavity // Phys. Rev. C. - 2002. Vol.65. - P. 044005

[57] T. Yamazaki and Y. Akaishi. $\left(\mathrm{K}-, \pi^{-}\right)$ production of nuclear $\overline{\mathrm{K}}$ bound states in proton-rich systems via $\Lambda \square$ doorways // Phys. Lett. B. - 2002. Vol.535. - P. 70.

[58] T. Yamazaki. A. Doté. and Y. Akaishi. Invariant-mass spectroscopy for condensed single- and double- $\overline{\mathrm{K}}$ nuclear clusters to be formed as residues in relativistic heavy-ion collisions // Phys. Lett. B. - 2004. Vol. 587. - P. 167.

[59] A. Doté. H. Horiuchi. Y. Akaishi. and T.Yamazaki. High-density Full-size image $(<1 \mathrm{~K})$ nuclear systems with isovector deformation // Phys. Lett. B. 2004. - Vol. 590. - P. 51.

[60] A. Doté. H. Horiuchi. Y. Akaishi. and T.Yamazaki. Kaonic nuclei studied based on a new framework of antisymmetrized molecular dynamics // Phys. Rev. C. - 2004. - Vol. 70. - P. 044313.

[61] T. Yamazaki and Y. Akaishi. Basic K- nuclear cluster, $\mathrm{K}-\mathrm{pp}$, and its enhanced formation in the $\mathrm{p}+\mathrm{p} \rightarrow \mathrm{K}++\mathrm{X}$ reaction // Phys. Rev. C. -2007 . - Vol. 76. P. 045201 .

[62] A. Doté. H. Horiuchi. Y. Akaishi. and T. Yamazaki. The Study of Deeply Bound Kaonic Nuclei with Antisymmetrized Molecular Dynamics // Prog. Theor. Phys. Suppl. - 2002. - Vol. 146. - P. 508.

[63] A. Doté and W. Weise. // Prog. Theor. Phys. Suppl. - 2007. - Vol. 168. - P. 593.

[64] A. Doté. T. Hyodo. and W. Weise. Study of Light Kaonic Nuclei with a Chiral SU(3)-Based KN Interaction // Nucl. Phys. A. - 2008. - Vol. 804. - P. 197.

[65] A. Doté. T. Hyodo. and W. Weise. Variational calculation of the ppK system based on chiral SU(3) dynamics // Phys. Rev. C. - 2009. - Vol. 79. - P. 014003.

[66] S. Wycech and A. M. Green. Variational calculations for K- few-nucleon systems // Phys. Rev. C. 2009. - Vol. 79. - P. 014001.

[67] A. Doté. T. Inoue. and T. Myo. J. Phys: Conf. Ser. - 2014. - Vol. 569. - P. 012084.

[68] Y. H. Li and S.S. Wu. // Chinese Phys. C. - 2009. - Vol. 33. - P. 76.

[69] N. V. Shevchenko. A. Gal. J. Mareš. Faddeev Calculation of a K-pp Quasibound State // Phys. Rev. Lett. - 2007. - Vol. 98. - P. 082301.

[70] N. V. Shevchenko. A. Gal. and J. Mareš. and J.Révai. $\mathrm{K}-\mathrm{NN}$ quasibound state and the $\mathrm{K}-\mathrm{N}$ interaction: Coupled-channels Faddeev calculations of the $\mathrm{K}-\mathrm{NN}-\pi \Sigma \mathrm{N}$ system // Phys. Rev. C. -2007 . - Vol. 76. P.044004. 
[71] Y. Ikeda and T. Sato. Strange dibaryon resonance in the $\mathrm{K}-\mathrm{NN}-\pi \mathrm{YN}$ system // Phys. Rev. C. - 2007. Vol.76. - P. 035203.

[72] Y. Ikeda and T. Sato. Resonance energy of the $\mathrm{K}-\mathrm{NN}-\pi \mathrm{YN}$ system // Phys. Rev. C. - 2009. - Vol. 79. P. 035201

[73] A. Martnez Torres. K. P. Khemchandani. and E.Oset. Solution to Faddeev equations with two-body experimental amplitudes as input and application to $\mathrm{JP}=1 / 2+, \mathrm{S}=0$ baryon resonances // Phys. Rev. C. - 2009. - Vol. 79. - P. 065207.

[74] Y. Ikeda. H. Kamano. and T. Sato. Energy Dependence of $\overline{\mathrm{K}} \mathrm{N}$ Interactions and Resonance Pole of Strange Dibaryons // Prog. Theor. Phys. - 2010. - Vol. 124. - P. 533.

[75] M. Bayar. J. Yamagata-Sekihara. and E. Oset. K-NN system with chiral dynamics // Phys. Rev. C. - 2011. - Vol. 84. - P. 015209.

[76] E. Oset. D. Jido. T. Sekihara. A. Martnez Torres. K. P. Khemchandani. M. Bayar and J. Yamagata-Sekihara. A new perspective on the Faddeev equations and the system from chiral dynamics and unitarity in coupledK-NN channels // Nucl. Phys. A. - 2012. - Vol. 881. - P. 127.

[77] M. Bayar and E. Oset. Improved fixed center approximation of the Faddeev equations for the the $\mathrm{K}$ NN system with $\mathrm{S}=0$ // Nucl. Phys. A. - 2012. - Vol. 883. - P.57.

[78] M. Bayar and E. Oset. The K-NN system revisited including absorption // Nucl. Phys. A. - 2013. - Vol. 914. - P. 349.

[79] S. Maeda. Y. Akaishi. and T. Yamazaki.Strong binding and shrinkage of single and double $\mathrm{K}$ nuclear systems $(\mathrm{K}-\mathrm{pp}, \mathrm{K}-\mathrm{ppn}, \mathrm{K}-\mathrm{K}-\mathrm{p}$ and $\mathrm{K}-\mathrm{K}-\mathrm{pp})$ predicted by Faddeev-Yakubovsky calculations // Proc. Jpn. Acad. Ser. B. - 2013. - Vol. 89. - P. 418.

[80] J. Révai and N. V. Shevchenko. Faddeev calculations of the $\mathrm{K}-\mathrm{NN}$ system with a chirally motivated $\mathrm{K}-\mathrm{N}$ interaction. II. The $\mathrm{K}-\mathrm{pp}$ quasibound state // Phys. Rev. C. - 2014. - Vol. 90. - P. 034004.

[81] R. Ya. Kezerashvili. Sh. M. Tsiklauri. I. N. Filikhin. V. M. Suslov. and B. Vlahovic. Three-body calculations for the $\mathrm{K}-\mathrm{pp}$ system within potential models // J. Phys. G: Nucl. Part. Phys. - 2003. Vol. 43. - P. 065104.

[82] N. Barnea. A. Gal. and E. Z. Liverts. Three-body calculations for the $\mathrm{K}-\mathrm{pp}$ system within potential models // Phys. Lett. B. - 2012. - Vol. 712. - P. 132.

[83] R. Ya. Kezerashvili and Sh. M. Tsiklauri. Investigation of the structure of the few body kaonic nuclei using the method of hyperspherical functions in momentum space // EPJ Web Conf. - 2014. - Vol. 81. - P. 02022.

[84] M. Agnello. G. Beer. L. Benussi et al.Evidence for a Kaon-Bound State $\mathrm{K}-\mathrm{pp}$ Produced in $\mathrm{K}$ - Absorption Reactions at Rest // Phys. Rev. Lett. - 2005. - Vol. 94. - P. 212303.
[85] J-PARC E15 Collaboration: T. Hashimoto. S.Ajimura. G. Beer. et al.Search for the deeply bound $\mathrm{K}-\mathrm{pp}$ state from the semi-inclusive forward-neutron spectrum in the in-flight $\mathrm{K}$ - reaction on helium-3 // Prog. Theor. Exp. Phys. - 2015. - P. 061D01.

[86] J-PARC E27 Collaboration: Y. Ichikawa. et al.Experiment to Search for a Nuclear Kaon Bound State K-pp // Prog. Theor. Exp. Phys. - 2014. - P. 101D03;

[87] Y. Ichikawa. et al.Observation of the "K- pp"like structure in the $\mathrm{d}(\pi+, \mathrm{K}+)$ reaction at $1.69 \mathrm{GeV} / \mathrm{c} / /$ Prog. Theor. Exp. Phys. - 2015. - P. 021 D01.

[88] HADES Collaboration: G. Agakishiev. et al.Partial Wave Analysis of the Reaction $\mathrm{p}(3.5 \mathrm{GeV})+\mathrm{p}->\mathrm{pK}+\Lambda$ to Search for the "ppK-" Bound State // Phys. Lett. B. - 2015. - Vol. 742. - P. 242.

[89] T. Yamazaki. et al.Indication of a Deeply Bound and Compact $\mathrm{K}-\mathrm{pp}$ State Formed in the $\mathrm{pp} \rightarrow \mathrm{p} \Lambda \mathrm{K}+$ Reaction at $2.85 \mathrm{GeV} / /$ Phys. Rev. Lett. - 2010. - Vol. 104. - P. 132502.

[90] T. Suzuki et al.Discovery of a strange tribaryon $\mathrm{S} 0(3115)$ in $4 \mathrm{He}(\mathrm{K}-$ stopped,p) reaction // Phys. Lett. B. - 2004. - Vol. 597. - P. 263.

[91] T. Suzuki et al.A search for deeply bound kaonic nuclear states// Nucl. Phys. A. - 2005. - Vol. 754. - P.375.

[92] M. Sato et al. Search for strange tribaryon states in the inclusive $4 \mathrm{He}(\mathrm{K}-$ stopped,p) reaction // Phys. Lett. B. - 2008. - Vol. 659. - P. 107.

[93] M. Sato. et al. // Int. J. Mod. Phys. A.- 2009. - Vol.24. - P. 442.

[94] T. Hyodoand W. Weise. Effective $\mathrm{K}-\mathrm{N}$ interaction based on chiral SU(3) dynamics // Phys. Rev. C. - 2008. - Vol. 77. - P. 035204 .

[95] R. B. Wiringa. R. A. Smith. and T.L. Ainsworth. Nucleon-nucleon potentials with and without $\Delta(1232)$ degrees of freedom // Phys. Rev. C. - 1984. - Vol. 29. - P. 1207.

[96] R. B. Wiringa. V. G. J. Stoks. and R. Schiavilla. Accurate nucleon-nucleon potential with charge-independence breaking // Phys. Rev. C. - 1995. Vol. 51. - P. 38.

[97] R. A. Malfliet and J. A. Tjon. Solution of the Faddeev equations for the triton problem using local two-particle interactions // Nucl. Phys. A. - 1969. - Vol. 127. - P. 161.

[98] R. Tamagaki.Superfluid State in Neutron Star Matter. I: Generalized Bogoliubov Transformation and Existence of 3P2 Gap at High Density // Prog. Theor. Phys. - 1970. - Vol. 44. - P. 905.

[99] D. R. Thompson. M. LeMere. Y. C. Tang. Systematic investigation of scattering problems with the resonating-group method // Nucl. Phys. A. - 1977. - Vol. 286. - P. 53.

[100] Y. Akaishi. K. S. Myint. and T. Yamazaki. Kaonic nuclear systems KbarN and KbarNN as decaying states// Proc. Jpn. Acad. Ser. B. - 2008. Vol.84. - P. 264. 